\title{
SIMULAÇÃO DA UNIFORMIDADE DA IRRIGAÇÃO DE SISTEMAS CONVENCIONAIS DE ASPERSÃO OPERANDO SOB DIFERENTES CONDIÇÕES DE VENTO
}

\author{
LESSANDRO C. FARIA ${ }^{1}$, ALBERTO COLOMBO ${ }^{2}$, HENRIQUE F. E. OLIVEIRA ${ }^{3}$, \\ GIULIANI DO PRADO ${ }^{4}$
}

\begin{abstract}
RESUMO: A uniformidade de aplicação de água é um importante fator a ser considerado na avaliação dos sistemas de irrigação por aspersão. A uniformidade em sistemas convencionais de aspersão depende de fatores como: o tipo de aspersor e suas condições operacionais, a disposição e o espaçamento entre aspersores no campo, e a velocidade e a direção do vento durante o período de aplicação de água. Tendo em visa que as complexas relações entre esses fatores e a uniformidade podem ser caracterizadas de forma mais eficiente com o auxílio da modelagem matemática, neste estudo, foram avaliadas simulações, fornecidas pelo modelo semiempírico de RICHARDS \& WEATHERHEAD (1993), da distribuição espacial da água aplicada pelo canhão PLONA-RL250, operando sob diferentes condições de vento. Valores de uniformidade de Christiansen (CUC), obtidos com dados de ensaios de campo e em simulações digitais, foram comparados, mostrando-se, por meio de diversos índices $\left(\mathrm{R}^{2}=0,73\right.$; desvio absoluto médio de $4,1 \%$ e índice de desempenho de 0,78), que o modelo semiempírico avaliado fornece estimativas adequadas do CUC de sistemas convencionais de irrigação. Demonstra-se que a modelagem matemática permite reduzir o esforço e o tempo requeridos em trabalhos de campo envolvendo a avaliação da distribuição de água de canhões hidráulicos operando sob condições de vento.
\end{abstract}

PALAVRAS-CHAVE: PLONA-RL250, canhão hidráulico, Richard \& Weatherhead.

\section{WATER APPLICATION UNIFORMITY SIMULATION OF SOLID SET SPRINKLER SYSTEMS OPERATING UNDER DIFFERENT WIND CONDITIONS}

\begin{abstract}
The water application uniformity is an important factor to be considered on the sprinkler irrigation system evaluation. Solid set sprinkler system water application uniformity depends on factors such as: sprinkler type and its operational conditions, sprinkler disposition and spacing on the field, and wind velocity and direction during water application periods. Considering that the complex relationships among these factors and uniformity are more efficiently characterized by employing mathematical modeling, this study aimed to evaluate simulations, provided by the RICHARDS \& WEATHERHEAD (1993) semi-empirical model of the spatial distribution of the water applied by a PLONA RL-250 gun type sprinkler operating under different wind conditions. Values of Christiansen coefficient of uniformity (CUC) based on in-field single sprinkler tests were compared to simulated values showing, through different evaluation indices $\left(\mathrm{R}^{2}=0.73\right.$; a $4.1 \%$ average absolute deviation, and a 0.78 performance index), that the evaluated semi-empirical model provides reliable estimates of CUC of solid set sprinkler systems. It is demonstrated that mathematical simulations can provide reduction on both the effort and the time required to perform field work related to spatial water distribution evaluations of single gun type sprinklers operating under windy conditions.
\end{abstract}

KEYWORDS: PLONA-RL250, gun type sprinkler, Richard \& Weatherhead.

\footnotetext{
${ }^{1}$ Eng ${ }^{\circ}$ Agrícola, Doutorando em Engenharia Agrícola, UFLA, Lavras - MG, lessandrofaria@ yahoo.com.br

${ }^{2}$ Engoㅡ Agrônomo, Ph.D., UFLA, Lavras - MG, acolombo@ufla.br

${ }^{3}$ Eng $^{0}$ Agrícola, Doutorando em Engenharia Agrícola, UFLA, Lavras - MG, henrique.ellias@ hotmail.com

${ }^{4}$ Eng ${ }^{0}$ Agrônomo, Prof. Doutor, UFLA, Lavras - MG, giulianip@bol.com.br

Recebido pelo Conselho Editorial em: 10-4-2008

Aprovado pelo Conselho Editorial em: 18-2-2009
} 


\section{INTRODUÇÃO}

Os sistemas de irrigação por aspersão devem aplicar água da maneira mais uniforme possível, visto que a desuniformidade de aplicação de água diminui o retorno econômico e aumenta o impacto ambiental da irrigação, em função da redução na produtividade das culturas irrigadas e do desperdício de água, de energia e de fertilizantes.

De acordo com MATEOS (1998), a distribuição da água aplicada dos sistemas de aspersão é um processo que passa por duas etapas: aplicação da água na superfície do solo ou da cultura e redistribuição da água aplicada no interior do solo. Apesar dos efeitos positivos da redistribuição da água no solo, que, em alguns casos, pode anular os efeitos da desuniformidade de aplicação (LI \& RAO, 2003), deve-se considerar, conforme enfatizado por FRIZZONE et al. (2007), que a uniformidade do teor de água do solo e a produtividade das culturas irrigadas são bastante dependentes da uniformidade com que a água é aplicada durante a primeira fase desse processo.

MATEOS (1998) classifica os diversos fatores que afetam a uniformidade da aplicação de água na superfície do solo de acordo com as seguintes categorias: (i) características do aspersor (modelo, número e tamanho de bocais, pressão de serviço e ângulo do jato do aspersor); (ii) distribuição do sistema (altura de instalação dos aspersores, espaçamento, tipo de arranjamento, declividade do terreno e variações de pressão); (iii) fatores climáticos (direção e velocidade do vento e evaporação das gotas), e (iv) fatores do manejo (inclinação das hastes dos aspersores, tempo de operação por posição e tipo de movimentação).

Ao avaliar o efeito de alguns desses fatores sobre a uniformidade de aplicação de água de sistemas convencionais de aspersão, equipados com canhões hidráulicos, AZEVEDO et al. (2000) observaram que a velocidade do vento foi o fator que mais influenciou na uniformidade de aplicação da água, seguida pela pressão de operação do aspersor, espaçamento entre aspersores na linha lateral, espaçamento entre linhas laterais, direção do vento em relação à linha lateral e velocidade de rotação do aspersor.

A teoria balística tem sido utilizada em diversos modelos matemáticos para a simulação da distribuição espacial da água aplicada por pequenos aspersores operando em diferentes condições de vento (CARRIÓN et al., 2001; PLAYAN et al., 2006). A adequação das simulações da distribuição de água de microaspersores, baseadas na teoria balística, do modelo SIRIAS de CARRIỎN et al. (2001), foi comprovada no trabalho de CONCEIÇÃO \& COELHO (2003).

Para o caso da simulação da distribuição da água aplicada por meio de canhões hidráulicos, operando em diferentes condições de vento, as dificuldades de se aplicar o modelo balístico fizeram com que diversos pesquisadores (GRANIER et al., 2003; PRADO, 2008; SMITH et al., 2008) optassem pela adoção do modelo semiempírico de RICHARDS \& WEATHERHEAD (1993). No entanto, estes estudos focam sua aplicabilidade na previsão da uniformidade de equipamentos autopropelidos de aspersão, sem considerar a aplicabilidade do modelo de RICHARDS \& WEATHERHEAD (1993) na previsão da uniformidade de aplicação de água de sistemas convencionais de aspersão dotados de canhões hidráulicos.

Dessa forma, visando a preencher essa lacuna, este estudo teve como objetivo avaliar a aplicabilidade do modelo semiempírico de RICHARDS \& WEATHERHEAD (1993) na previsão da uniformidade de aplicação de água do canhão PLONA-RL250, operando sob diferentes condições de vento, em sistemas convencionais de irrigação por aspersão.

\section{MATERIAL E MÉTODOS}

O trabalho foi realizado a partir de dados coletados em 53 ensaios de distribuição de água do canhão PLONA-RL250, que foram realizados no Centro Federal de Educação Tecnológica (CEFET), Uberaba - MG, sob diferentes condições de velocidade de vento, e combinações de bocais e pressões de serviço mostradas na Tabela 1. Nos ensaios de distribuição de água, com duração aproximada de uma hora, foi utilizada malha de coletores plásticos de $8 \mathrm{~cm}$ de diâmetro, regularmente espaçados de $6 \mathrm{~m}$ ao redor do aspersor. A velocidade e a direção do vento durante esses ensaios foram registradas em intervalos regulares de 5 minutos, com o auxílio de anemômetro, modelo Weather MonitorII da Davis, instalado à altura de $2 \mathrm{~m}$ do solo. 
TABELA 1. Velocidade média do vento e combinações de bocais e pressão de serviço registradas nos 53 ensaios de distribuição de água do canhão PLONA-RL250. Average wind speed and nozzle pressure combinations during the 53 PLONA-RL250 catch can tests held at the CEFET - Uberaba - MG, Brazil.

\begin{tabular}{|c|c|c|c|c|c|c|c|c|c|c|}
\hline \multirow{3}{*}{$\begin{array}{l}\text { Bocal } \\
\text { Auxiliar } \\
\text { (mm) }\end{array}$} & \multicolumn{10}{|c|}{ Pressão de Serviço (kPa) } \\
\hline & 392 & 490 & 392 & 490 & 392 & 490 & 392 & 490 & 392 & 490 \\
\hline & \multicolumn{10}{|c|}{ Velocidade média do vento durante cada ensaio $\left(\mathrm{m} \mathrm{s}^{-1}\right)$} \\
\hline \multirow{2}{*}{7} & 4,96 & 4,72 & 2,95 & 3,13 & 5,32 & 5,83 & & & & \\
\hline & 2,96 & 3,45 & & 2,84 & 4,74 & 4,64 & & & & \\
\hline \multirow{3}{*}{6} & 1,76 & & 2,79 & 1,83 & 3,57 & 3,39 & 2,43 & 2,71 & & 2,87 \\
\hline & 0,97 & & 2,61 & & 2,54 & 1,34 & 1,73 & 2,04 & & 2,74 \\
\hline & 0,96 & & & & 2,01 & & 1,00 & & & \\
\hline \multirow{3}{*}{5} & 4,11 & 3,92 & 1,82 & 1,72 & 2,27 & 4,13 & & & & \\
\hline & 1,71 & 1,61 & 1,39 & 1,56 & & 2,48 & & & & \\
\hline & & & 1,05 & & & & & & & \\
\hline \multirow{3}{*}{0} & 4,13 & 4,00 & 2,24 & 2,25 & 2,03 & 2,39 & & & & \\
\hline & 1,77 & 1,15 & 1,58 & 1,90 & & 1,17 & & & & \\
\hline & & & & 0,99 & & & & & & \\
\hline $\begin{array}{c}\text { Bocal } \\
\text { Principal }\end{array}$ & \multicolumn{2}{|c|}{$14 \mathrm{~mm}$} & \multicolumn{2}{|c|}{$16 \mathrm{~mm}$} & \multicolumn{2}{|c|}{$18 \mathrm{~mm}$} & \multicolumn{2}{|c|}{$20 \mathrm{~mm}$} & \multicolumn{2}{|c|}{$22 \mathrm{~mm}$} \\
\hline
\end{tabular}

Os volumes de água coletados ao final de cada ensaio foram convertidos em valores de intensidade de precipitação $\left(\mathrm{mm} \mathrm{h}^{-1}\right)$ e registrados em matriz "A", de 14 x 14 . O sistema de eixos $(\mathrm{X}, \mathrm{Y})$ adotado nos ensaios de campo para a identificação das coordenadas dos coletores representados na matriz "A", e a notação horária de registro do ângulo $\Phi$, que descreve o sentido do vento em relação às linhas de coletores de água utilizados nos ensaios de campo, são mostrados na Figura 1.

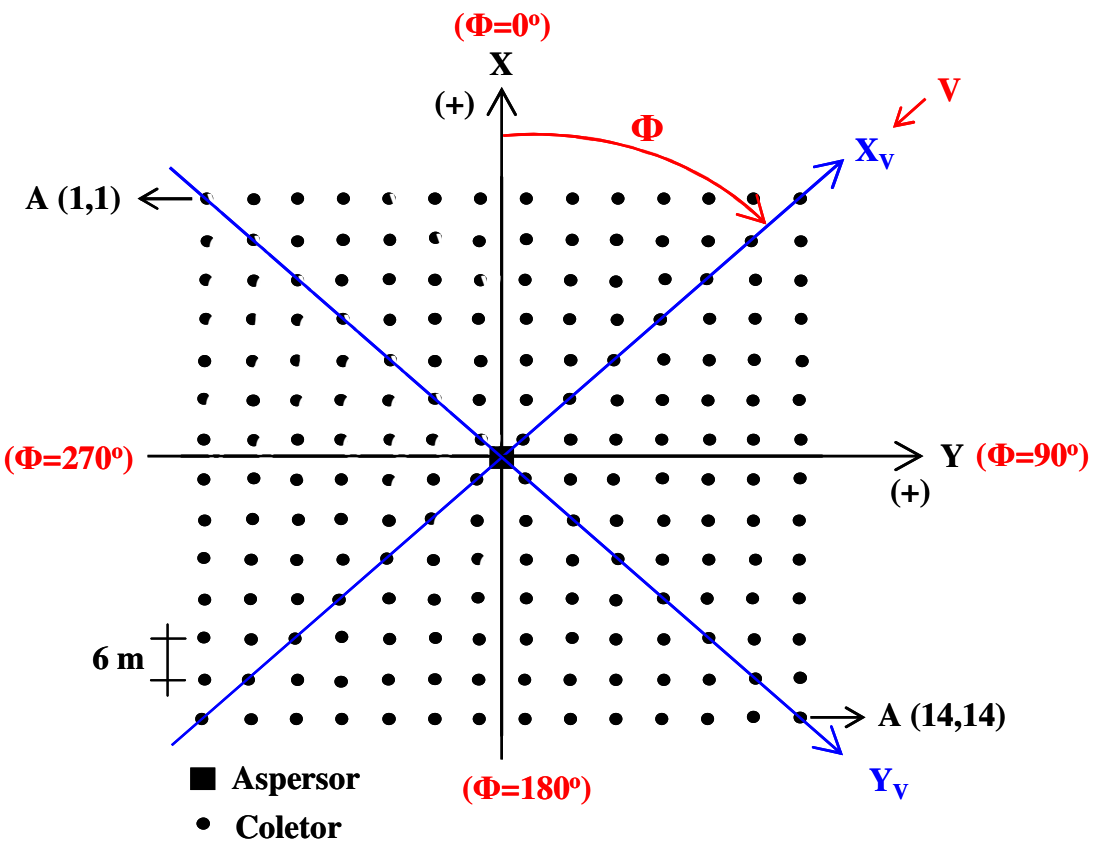

FIGURA 1. Esquema do sistema de eixos $(\mathrm{X}, \mathrm{Y})$ utilizado nos ensaios de campo e do ângulo $\Phi$ da direção do vento, que determina o posicionamento do sistema de eixos $\left(\mathrm{X}_{\mathrm{V}}, \mathrm{Y}_{\mathrm{V}}\right)$ utilizado no modelo de RICHARDS \& WEATHERHEAD (1993). Sketch of the $(\mathrm{X}, \mathrm{Y})$ axis system used on field test showing the wind direction $\Phi$ angle that is used for positioning the $\left(X_{V}, Y_{V}\right)$ of the RICHARDS \& WEATHERHEAD (1993) model. 
As simulações dos valores de precipitação, correspondentes às mesmas condições operacionais e de vento observadas durante os ensaios de campo realizados com o PLONA-RL250, foram realizadas com base no modelo semiempírico de RICHARDS \& WEATHERHEAD (1993). Nessas simulações, os seguintes dados de entrada foram considerados:

(i) velocidade média $\left(\mathrm{V}\right.$, em $\left.\mathrm{m} \mathrm{s}^{-1}\right)$ e direção média do vento $(\Phi$, em graus), conforme registradas durante o ensaio de campo a ser simulado;

(ii) coordenadas $(\mathrm{x}, \mathrm{y})$ dos coletores, tomadas no sistema de eixos orientados dos ensaios de campo;

(iii) ângulo de saída do jato de água do canhão PLONA-RL250 em relação ao plano horizontal $\left(\mathrm{e}=24^{\mathrm{o}}\right)$;

(iv) valores, fornecidos pelo aplicativo computacional SimulaSoft (PRADO, 2004), do raio de alcance $(\mathrm{R}$, em $\mathrm{m})$ e de intensidades de precipitação $\left(\mathrm{I}(\mathrm{r} / \mathrm{R})_{0}\right.$, em $\left.\mathrm{mm} \mathrm{h}^{-1}\right)$ em 20 diferentes distâncias do aspersor $(\mathrm{r}$, em $\mathrm{m})$, correspondentes à frações do raio de alcance $(\mathrm{r} / \mathrm{R})$, variando desde $\mathrm{r} / \mathrm{R}=$ 0,025 até $r / R=0,975$, em intervalos constantes de 0,05 do valor do raio de alcance do canhão PLONA-RL250, operando, na ausência de vento, nas mesmas condições de pressão e diâmetro de bocal (is) observadas durante o ensaio de campo a ser simulado;

(v) valores, ajustados para o aspersor PLONA-RL250, por FARIA (2008), das constantes empíricas do modelo de RICHARDS \& WEATHERHEAD (1993), ponderadas pelo raio de alcance sem vento $\left(\mathrm{A} / \mathrm{R}=0,0362 \mathrm{~s} \mathrm{~m}^{-1} ; \mathrm{B} / \mathrm{R}=0,0131 \mathrm{~s} \mathrm{~m}^{-1} ; \mathrm{C} / \mathrm{R}=-0,0097 \mathrm{~s} \mathrm{~m}^{-1} ; \mathrm{D} / \mathrm{R}=0,0026 \mathrm{~s} \mathrm{~m}^{-1}\right.$; $\mathrm{E} / \mathrm{R}=0,4003 \mathrm{~s} \mathrm{~m}^{-1}$ e $\left.\mathrm{F} / \mathrm{R}=-0,3361 \mathrm{~s} \mathrm{~m}^{-1}\right)$.

Os dados de entrada foram processados, por meio de rotina desenvolvida em Visual Basic para Aplicativos (VBA) da planilha eletrônica Excel, de acordo com os seguintes passos:

(i) cálculo, com base no valor do raio de alcance sem vento $(\mathrm{R})$, do valor não ponderado $(\mathrm{A}=(\mathrm{A} / \mathrm{R}) \cdot \mathrm{R} \ldots, \quad \mathrm{F}=(\mathrm{F} / \mathrm{R}) \cdot \mathrm{R})$ das constantes empíricas do modelo de RICHARDS \& WEATHERHEAD (1993).

(ii) conversão, por meio das eqs. (1) e (2), das coordenadas (x,y) da posição do coletor, no sistema de eixos dos ensaios de campo, para as coordenadas $\left(\mathrm{x}_{\mathrm{v}}, \mathrm{y}_{\mathrm{v}}\right)$ do sistema de eixos orientado contra o sentido do vento definido no modelo de RICHARDS \& WEATHERHEAD (1993):

$$
\begin{aligned}
& \mathrm{x}_{\mathrm{v}}=\mathrm{x} \cos (\Phi)+\mathrm{y} \operatorname{sen}(\Phi) \\
& \mathrm{y}_{\mathrm{v}}=\mathrm{y} \cos (\Phi)+\mathrm{x} \operatorname{sen}(\Phi)
\end{aligned}
$$

(iii) cálculo, por meio de método iterativo, das coordenadas $\left(\mathrm{x}_{\mathrm{v} 0}, \mathrm{y}_{\mathrm{v} 0}\right)$ do ponto de impacto sem vento que resultam em um ponto de impacto com vento coincidente com a posição do coletor em questão, que tem coordenadas $\left(\mathrm{x}_{\mathrm{v}}, \mathrm{y}_{\mathrm{v}}\right)$. Esse processo iterativo tem início com $\mathrm{x}_{\mathrm{v} 0}=\mathrm{x}_{\mathrm{v}} \mathrm{e} \mathrm{y}_{\mathrm{v} 0}=\mathrm{y}_{\mathrm{v}}$, terminando quando o valor absoluto das correções das estimativas de $\mathrm{x}_{\mathrm{v} 0}$ e $\mathrm{y}_{\mathrm{v} 0}$ é menor que $0,001 \mathrm{~m}$. Para acelerar a convergência, o método de Newton, descrito por BURDEN \& FAIRES (2003), foi aplicado ao seguinte sistema de equações simultâneas, dadas no modelo de RICHARDS \& WEATHERHEAD (1993), para descrever a relação entre as coordenadas do ponto de impacto na ausência de vento $\left(\mathrm{x}_{\mathrm{v} 0}, \mathrm{y}_{\mathrm{v} 0}\right)$ e a coordenada do ponto de impacto na presença de vento $\left(\mathrm{x}_{\mathrm{v}}, \mathrm{y}_{\mathrm{v}}\right)$ :

$$
\begin{aligned}
& x_{v}=x_{v 0}-\left[A+B(r / R)+C(r / R)^{2}\right] V-\left[D(r / R)+E(r / R)^{2}+\ldots\right. \\
& \left.F(r / R)^{3}\right] V \sqrt{\operatorname{sen}^{2} e\left(\frac{x_{v 0}}{r}\right)^{2}+\left(\frac{y_{v 0}}{r}\right)^{2}} \frac{x_{v 0}}{r} \\
& y_{v}=y_{v 0}-\left[D(r / R)+E(r / R)^{2}+F(r / R)^{3}\right] V \sqrt{\operatorname{sen}^{2} e\left(\frac{x_{v 0}}{r}\right)^{2}+\left(\frac{y_{v 0}}{r}\right)^{2}} \frac{y_{v 0}}{r}
\end{aligned}
$$

em que, 
$\mathrm{x}_{\mathrm{v}}, \mathrm{y}_{\mathrm{v}}$ - coordenadas do ponto de impacto da água em condições de vento, $\mathrm{m}$;

$\mathrm{x}_{\mathrm{v} 0}, \mathrm{y}_{\mathrm{v} 0}$ - coordenadas do ponto de impacto da água na ausência de vento, $\mathrm{m}$;

$\mathrm{r}=\left(\mathrm{x}_{\mathrm{v} 0}{ }^{2}+\mathrm{y}_{\mathrm{v} 0}{ }^{2}\right)^{1 / 2}$ - distância radial do aspersor ao ponto de impacto sem vento, $\mathrm{m}$;

$\mathrm{R}$ - raio de alcance do aspersor PLONA-RL250 operando na ausência de vento, m;

$\mathrm{V}$ - velocidade do vento, $\mathrm{m} \mathrm{s}^{-1}$;

e - ângulo de saída do jato de água em relação ao plano horizontal, graus, e

A, B, C, D, E e F - constantes empíricas do modelo de RICHARDS \& WEATHERHEAD, $\mathrm{m}$ por $\mathrm{m} \mathrm{s}^{-1}$.

(iv) cálculo da taxa da aplicação, $\mathrm{I}(\mathrm{r} / \mathrm{R})_{0}$, na ausência de vento, no ponto de coordenadas $\mathrm{X}_{\mathrm{v} 0} \mathrm{e}$ $\mathrm{y}_{\mathrm{v} 0}$, que corresponde a distância radial $\mathrm{r}=\left(\mathrm{x}_{\mathrm{v} 0}{ }^{2}+\mathrm{y}_{\mathrm{v} 0}{ }^{2}\right)^{1 / 2}$ do perfil radial de aplicação de água;

(v) cálculo da taxa da aplicação $I_{v}$, no ponto de coordenadas $x_{v}, y_{v}$, por meio da eq.(5):

$$
I_{v}=\frac{I(r / R)_{0}}{\left(\frac{\partial x_{v}}{\partial x_{v 0}} \frac{\partial y_{v}}{\partial y_{v 0}}\right)}
$$

em que,

$\mathrm{I}_{\mathrm{V}}$ - intensidade de aplicação de água em $\mathrm{x}_{\mathrm{v}}, \mathrm{y}_{\mathrm{v}}$ (condições de vento, $\mathrm{mm} \mathrm{h}^{-1}$ );

$\mathrm{I}(\mathrm{r} / \mathrm{R})_{0}$ - intensidade de aplicação de água em $\mathrm{x}_{\mathrm{v} 0}, \mathrm{y}_{\mathrm{v} 0}$, (baseada na curva de aplicação de água do canhão PLONA-RL250 operando na ausência de vento, $\mathrm{mm} \mathrm{h}^{-1}$ ), e

$\frac{\partial x_{v}}{\partial x_{v 0}} e \frac{\partial y_{v}}{\partial y_{v 0}}-$ obtidos diferenciando-se as eqs. (3) e (4) em relação a $x_{v 0}$ e $y_{v 0}$.

(vi) execução dos passos (ii) até (v) para todos os coletores da malha dos ensaios de campo.

A uniformidade em sistemas convencionais de irrigação, operando com o aspersor PLONARL250, sob as mesmas condições observadas nos ensaios de campo, foi obtida pela sobreposição das matrizes contendo os valores de intensidade de precipitação ao redor desse aspersor. No processo de sobreposição, foram considerados apenas arranjos quadrados entre aspersores com espaçamentos múltiplos de $6 \mathrm{~m}$, variando desde $18 \mathrm{~m}$ até um valor não superior a $70 \%$ do diâmetro molhado do PLONA-RL250 observado na ausência de vento.

A adequação com que as simulações realizadas com modelo ajustado de RICHARDS \& WEATHERHEAD (1993) permitem prever valores de CUC, em sistemas convencionais de aspersão operando sob condições de vento, foi avaliada seguindo as recomendações MONTEIRO et al. (2001), para determinar o desvio absoluto médio e o valor do coeficiente de determinação $\left(\mathrm{R}^{2}\right)$ da reta ajustada aos pares (simulados e observados) de valores de CUC; e de CONCEIÇÃO \& COELHO (2003), para determinar o índice de confiança de desempenho (c) de CAMARGO \& SENTELHAS (1997), que é obtido pela multiplicação do coeficiente de correlação (r) pelo índice de Willmott (d), conforme apresentado nas expressões abaixo:

$\mathrm{c}=\mathrm{rd}$

com:

$$
\mathrm{d}=1-\left[\sum\left(\mathrm{P}_{\mathrm{i}}-\mathrm{O}_{\mathrm{i}}\right)^{2} / \sum\left(\left|\mathrm{P}_{\mathrm{i}}-\overline{\mathrm{O}}\right|+\left|\mathrm{O}_{\mathrm{i}}-\overline{\mathrm{O}}\right|\right)^{2}\right]
$$

em que,

$\mathrm{Pi}$-valor estimado;

Oi - valor observado, e

$\overline{\mathrm{O}}$ - média dos valores observados. 
O índice de confiança de desempenho foi avaliado segundo escala proposta por CAMARGO \& SENTELHAS (1997): Ótimo ( $c>0,85)$; Muito Bom $(0,76 \leq \mathrm{c} \leq 0,85)$; Bom $(0,66 \leq \mathrm{c} \leq 0,75)$; Mediano $(0,61 \leq \mathrm{c} \leq 0,65)$; Sofrível $(0,51 \leq \mathrm{c} \leq 0,60)$; Mau $(0,41 \leq \mathrm{c} \leq 0,50)$, e Péssimo $(\mathrm{c} \leq 0,40)$.

\section{RESULTADOS E DISCUSSÃO}

A relação entre 319 valores de CUC gerados a partir de simulações baseadas no modelo RICHARDS \& WEATHERHEAD (1993), ajustado ao aspersor PLONA-RL250, e 319 valores de CUC obtidos a partir de ensaios de campo, é mostrada na Figura 2. Os pares de valores de CUC apresentados na Figura 2 estão bem concentrados ao redor da reta 1:1. A adequação dessa concentração ao redor da linha 1:1 é confirmada pelo valor do índice de confiança dos valores simulados $(\mathrm{c}=0,85 \times 0,91=0,78)$ que, de acordo com a escala de CAMARGO \& SENTELHAS (1997), corresponde à classificação do tipo Muito Bom $(0,76 \leq \mathrm{c} \leq 0,85)$.

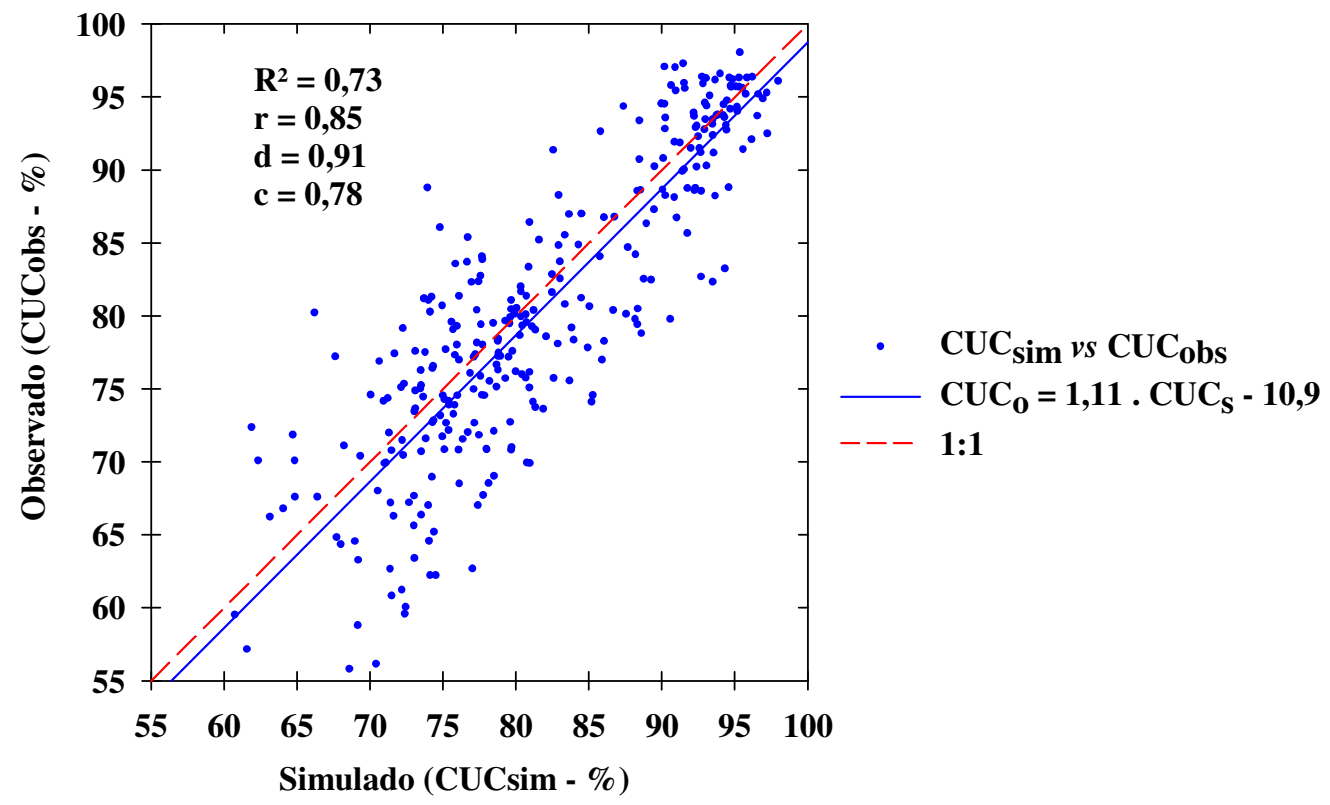

FIGURA 2. Valores de Coeficiente de Uniformidade de Christiansen obtidos a partir de simulações $\left(\mathrm{CUC}_{\mathrm{sim}}\right)$ e obtidos a partir de ensaios de campo $\left(\mathrm{CUC}_{\mathrm{obs}}\right)$. Christiansen Uniformity Coefficient values based on simulations $\left(\mathrm{CUC}_{\mathrm{sim}}\right)$ versus values based on field tests $\left(\mathbf{C U C} \mathbf{C}_{\text {obs }}\right)$.

Comparando-se a reta 1:1 com a linha do ajuste linear, verifica-se que, na região de interesse prático (CUC > 60\%), o modelo ajustado de RICHARDS \& WEATHERHEAD (1993) tende a produzir valores de CUC superiores aos observados nos ensaios de campo. O coeficiente de determinação $\left(R^{2}=0,73\right)$ do ajuste linear, entre valores simulados e observados de CUC, apresenta valor compatível com a faixa de valores de coeficiente de determinação obtidos por MONTERO et al. (2001), em estudo de avaliação do modelo SIRIAS $\left(\mathrm{R}^{2}\right.$ de 0,$51 ; 0,75 ; 0,85$ e 0,81 para os espaçamentos de, respectivamente, 12x12; 12x18; 18x18 e 18x16 m).

Na Figura 3, são apresentados 319 valores absolutos da diferença entre valores de CUC, expressos em percentagem, oriundos de simulações e de ensaios de campo. Nessa figura, pode-se observar que o valor médio das diferenças absolutas $(4,1 \%)$, representado pela linha vermelha, situa-se abaixo do limite de 5\%, que, segundo MONTERO et al. (2001), caracteriza desempenho classificado como "Muito Bom" na previsão de valores de CUC de sistemas convencionais de aspersão baseada em ensaios de campo de aspersores isolados. 


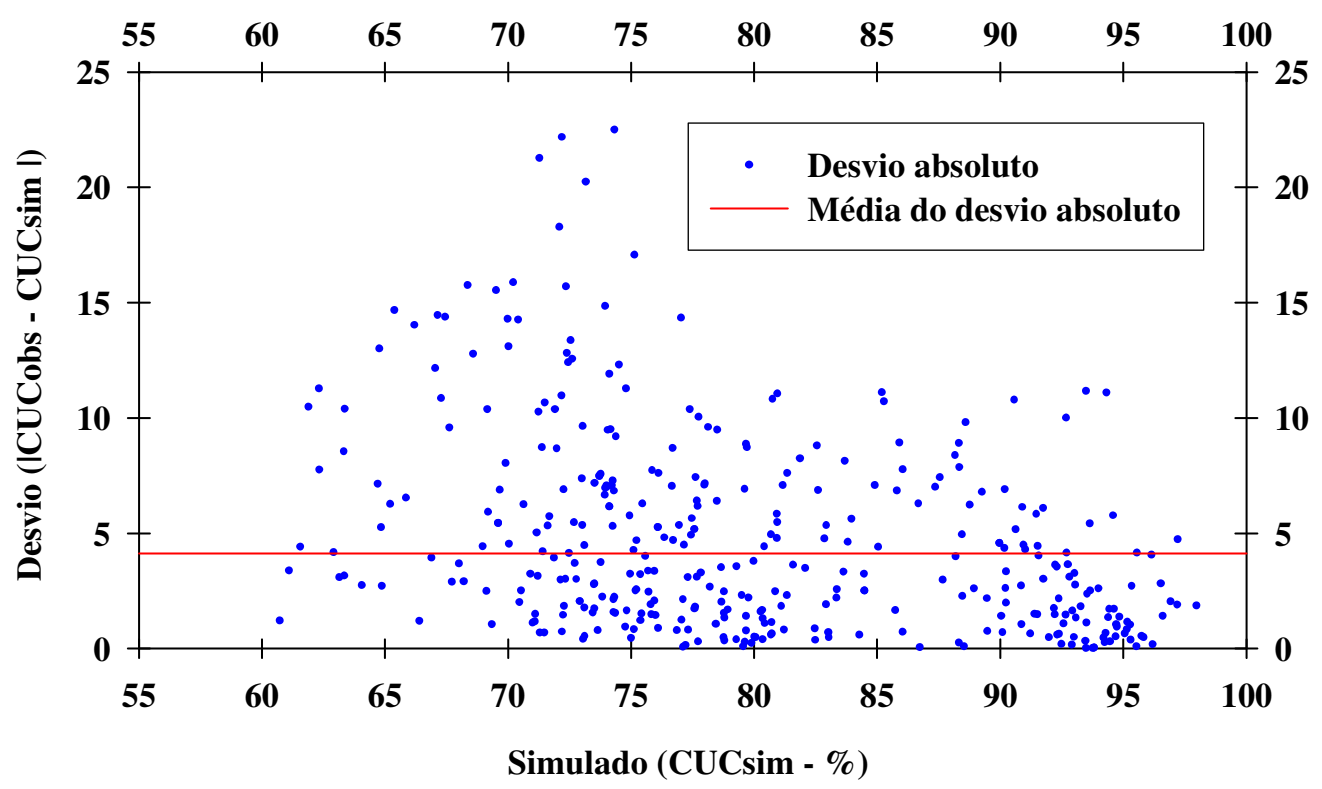

FIGURA 3. Diferenças absolutas entre estimativas de CUC obtidas com dados de ensaios de campo $\left(\mathrm{CUC}_{\mathrm{obs}}-\%\right)$ e com dados de ensaios simulados $\left(\mathrm{CUC}_{\text {sim }^{-}} \%\right)$. Absolute differences among CUC estimates based on field tests data $\left(\mathrm{CUC}_{\mathrm{obs}}\right)$ and simulated data $\left(\mathrm{CUC}_{\left.\text {sim- }^{-} \%\right)}\right.$.

A análise do desempenho do modelo ajustado não pode deixar de considerar que as diferenças nas estimativas de CUC encontradas neste trabalho não refletem apenas as deficiências do modelo ajustado, mas também refletem a dificuldade de se obter, ao longo do período de duração dos ensaios de campo, o mesmo grau de estabilidade nos valores dos parâmetros climáticos considerados nas simulações. $\mathrm{O}$ aumento da amplitude dos desvios correspondente aos menores valores de CUC, que é mostrado na Figura 3, indica que as estimativas que resultam em valores mais reduzidos de CUC, são mais suscetíveis às instabilidades dos fatores climáticos. Esse aspecto foi também enfatizado por SOLOMON (1979), ao demonstrar que, mesmo em condições de ventos fracos, os espaçamentos entre aspersores que resultam em reduzido grau de sobreposição da água aplicada por aspersores adjacentes e, consequentemente, valores menores de CUC, apresentam, em função das pequenas variações nas condições climáticas observadas durante os ensaios de campo, maior incerteza nos valores de CUC (desvio-padrão de $2 \%$ para CUC perto de $90 \%$, desvio-padrão de $4 \%$ para CUC próximo de $80 \%$ e desvio-padrão de $6 \%$ para CUC perto de $70 \%$ ).

$\mathrm{Na}$ Figura 4, é possível comparar, em diferentes espaçamentos entre aspersores dispostos em arranjos quadrados, estimativas de CUC obtidas por meio de ensaios de campo e simulações. As condições operacionais dos ensaios mostrados nessa figura (valores à direita) são as mesmas observadas nos ensaios que foram utilizados por FARIA (2008), para ajustar os valores dos parâmetros empíricos do modelo de RICHARDS \& WEATHERHEAD (1993).

$\mathrm{Na}$ Figura 4, a linha pontilhada vertical indica o espaçamento máximo entre aspersores, que, na ausência de vento, permite o recobrimento total da área entre quatro aspersores adjacentes. Pode-se observar, com o auxílio da linha pontilhada, o fato de que, mesmo em condições otimizadas de ajuste dos parâmetros empíricos do modelo de RICHARDS \& WEATHERHEAD (1993), a redução no grau de sobreposição das áreas molhadas por aspersores adjacentes é acompanhada por acréscimo nas diferenças entre as estimativas de CUC. 


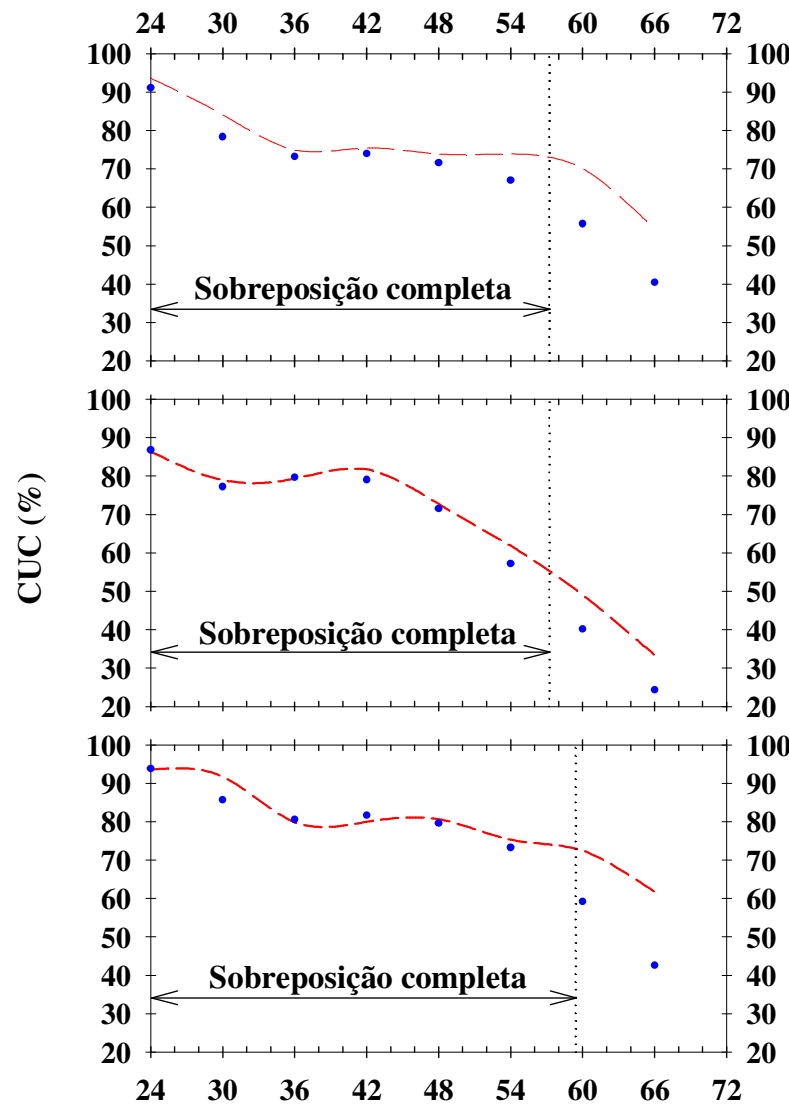

Espaçamento entre aspersores (m) (a)

Bocais: 16 x $6 \mathrm{~mm}$

Pressão: 490 kPa

Raio: $40,50 \mathrm{~m}$

Vazão: 23,32 m $^{3} / \mathrm{h}$

Vel. vento: $1,83 \mathrm{~m} / \mathrm{s}$

Dir. vento: $\mathbf{2 8}^{\circ}$

\section{(b)}

Bocais: 16 x $0 \mathrm{~mm}$

Pressão: 490 kPa

Raio: $40,65 \mathrm{~m}$

Vazão: $20,51 \mathrm{~m}^{3} / \mathrm{h}$

Vel. vento: $2,25 \mathrm{~m} / \mathrm{s}$

Dir. vento: $353^{\circ}$

(c)

Bocais: $18 \times 5$ mm

Pressão: 490 kPa

Raio: $42,00 \mathrm{~m}$

Vazão: $28,43 \mathrm{~m}^{3} / \mathrm{h}$

Vel. vento: $4,13 \mathrm{~m} / \mathrm{s}$

Dir. vento: $107,3^{\circ}$

CUCsim - simulado

CUCobs - observado

Sobreposição completa

FIGURA 4. Estimativas do Coeficiente de Uniformidade de Christiansen (CUC em \%), em função do espaçamento dos aspersores, geradas com dados simulados $\left(\mathrm{CUC}_{\mathrm{sim}}\right.$ - linhas) e com dados de ensaios de campo $\left(\mathrm{CUC}_{\mathrm{obs}}\right.$ - pontos). Christiansen Uniformity Coefficient (CUC em \%), as a function of sprinkler spacing, generated by simulated dada $\left(\mathrm{CUC}_{\text {sim }}\right.$ - lines $)$ and by field observed data $\left(\mathrm{CUC}_{\mathrm{obs}}\right.$ - points $)$.

Apesar das diferenças nos valores absolutos das estimativas de CUC, mostradas na Figura 4, observa-se que o comportamento da uniformidade de aplicação, em função do aumento do espaçamento entre aspersores, determinado com base nos dados de ensaios de campo, foi adequadamente representado nas três condições simuladas (Figuras 4a, 4b e 4c).

\section{CONCLUSÕES}

Nas situações de interesse prático, nas quais o grau de sobreposição das áreas molhadas por aspersores adjacentes é elevado, o modelo empírico de RICHARDS \& WEATHERHEAD (1993) foi capaz de fornecer estimativas adequadas do coeficiente de uniformidade da aplicação de água de Christiansen, de sistemas convencionais de irrigação operando com o canhão PLONA-RL250 sob diferentes condições de vento.

O bom desempenho do modelo ajustado permite inferir que o trabalho de campo requerido para produzir dados de uniformidade de aplicação de água de canhões hidráulicos, operando em diferentes condições de vento, pode ser bastante simplificado pela utilização do modelo semiempírico de RICHARDS \& WEATHERHEAD (1993). 


\section{REFERÊNCIAS}

AZEVEDO, H.J.; BERNARDO, S.; RAMOS, M.M.; SEDIYAMA, G.C.; CECON, P.R. Influência de fatores climáticos e operacionais sobre a uniformidade de distribuição de água, em um sistema de irrigação por aspersão de alta pressão. Revista Brasileira de Engenharia Agrícola e Ambiental, Campina Grande, v.4, n.2, p.152-158, 2000.

BURDEN, R.L.; FAIRES, J. D. Análise numérica. São Paulo: Thomson Learning, 2003. 736 p.

CAMARGO, A.P. de; SENTELHAS, P.C. Avaliação do desempenho de diferentes métodos de estimativa da evapotranspiração potencial no Estado de São Paulo, Brasil. Revista Brasileira de Agrometeorologia, Santa Maria, v.5, n.1, p.89-97, 1997.

CARRIÓN, P.; TARJUELO, J.M.; MOTERO, J. SIRIAS: a simulation model for sprinkler irrigation. Part I: Description of model. Irrigation Science, New York, v.20, n.2, p.73-84, 2001.

FARIA, L.C. Simulação da uniformidade de aplicação de água em sistemas convencionais de irrigação operando sob diferentes condições de vento. 2008. 76 f. Dissertação (Mestrado em Engenharia de Água e Solo) - Universidade Federal de Lavras, Lavras, 2008.

CONCEIÇÃO, M. A. F.; COELHO, R. D. Simulating wind effect on microsprinkler water distribution. Scientia Agricola, Piracicaba, v.60, n.2, p.205-209, 2003.

FRIZZONE, J.A.; REZENDE, R.; GONÇALVES, A.C.A.; HEBEL JÚNIOR, A. Produtividade do feijoeiro sob diferentes uniformidades de distribuição de água na superfície e na subsuperfície do solo. Engenharia Agrícola, Jaboticabal, v.27, n.2, p.414-425, 2007.

GRANIER, J.; MOLLE, B.; DEUMIER, J. M. IRRIPARC-Part 1: Modeling spatial water distribution under a sprinkler in windy conditions. In: EUROPEN REGIONAL CONFERENCE OF THE INTERNATIONAL COMISSION ON IRRIGATION AND DRAINAGE, 20., 2003, Montpellier. Proceedings... Montpellier: AFEID, 2003. p.14-19.

LI, J.; RAO, M. Field evaluation of crop yield as affected by nonuniformity of sprinkler-applied water and fertilizer. Agricultural Water Management, Amsterdam, v.76, n.3, p.1-13, 2003.

MATEOS, L. Assessing whole-field uniformity of stationary sprinkler irrigation systems. Irrigation Science, New York, v.18, n. 2, p.73-81, 1998.

MONTERO, J.; TARJUELO, J.M.; CARRIÓN, P. SIRIAS: a simulation model for sprinkler irrigation. Part II: Calibration and validation of the model. Irrigation Science, New York, v.20, n.2, p.85-98, 2001.

PRADO, G. Aplicativo computacional para simulação da distribuição de água pelo aspersor PLONA - RL300 em sistemas autopropelidos de irrigação. 2004. 86 f. Dissertação (Mestrado em Irrigação e Drenagem) - Universidade Federal de Lavras, Lavras, 2004.

PRADO, G. Modelagem da aplicação de água com canhões hidráulicos sob diferentes condições de vento. 2008. $121 \mathrm{f}$. Tese (Doutorado em Engenharia de Água e Solo) - Universidade Federal de Lavras, Lavras, 2008.

PLAYAN, E.; ZAPATA, N.; FACI, J.M.; TOLOSA, D.; LACUERVA, J.L.; PELEGRI, J.; SALVADOR, R.; SANCHES, I.; LAFITA, A. Assessing sprinkler irrigation uniformity using a ballistic simulation model. Agricultural Water Management, Amsterdam, v.84, n.1, p.89-100, 2006.

RICHARDS, P.J.; WEATHERHEAD, E. K. Prediction of raingun application patterns in windy conditions. Journal of Agricultural Engineering Research, Amsterdam, v.54, n.4, p.281-291, 1993.

SMITH, R.J.; GILLIES, M.H.; NEWELL, G.; FOLEY, J.P. A decision support model for travelling gun irrigation machines. Biosystems Engineering, Amsterdam, v.100, n.1, p.126-136, 2008.

SOLOMON, K. Variability of sprinkler coefficient of uniformity test results. Transactions of the American Society of Agricultural Engineers, St. Joseph, v.22, n.5, p.1.078-80-1.086, 1979. 